\title{
Theory of powdery rubber wear
}

\author{
B N J Persson \\ IFF, FZ-Jülich, 52425 Jülich, Germany \\ Received 17 January 2009, in final form 18 June 2009 \\ Published 30 October 2009 \\ Online at stacks.iop.org/JPhysCM/21/485001
}

\begin{abstract}
Rubber wear typically involves the removal of small rubber particles from the rubber surface. On surfaces with not too sharp roughness, e.g. most road surfaces, this involves (slow) crack propagation. In this paper I shall present a theory of mild rubber wear. I shall derive the distribution of wear particle sizes $\Phi(D)$, which is in excellent agreement with experiment. I shall also show that the calculated wear rate is consistent with experimental data for tire tread block wear.
\end{abstract}

(Some figures in this article are in colour only in the electronic version)

\section{Introduction}

Rubber wear involves extremely complex physical processes, but is a topic of great practical importance, e.g. tire wear [1-14]. When a rubber block is sliding on a hard rough substrate, rubber particles will continuously be removed from the block $^{1,2}$. This wear process involves two different steps, namely (a) nucleation of crack-like defects and (b) propagation of the cracks, resulting in the detachment of rubber particles. The detached rubber particles are usually very small, e.g., tire tread wear on road surfaces produces particles with sizes $D \sim 1-100 \mu \mathrm{m}$. Most of the particles are in the micrometer range but the largest particles give the largest wear volume. In figure 1 I show the surface morphology of a new (not used) rubber tread block (top), and of a used tread block (bottom). Note the strong roughness of the used tread block on the length scale of the order of $10 \mu \mathrm{m}$. At some places sharp edges occur as expected if rubber particles have been removed by crack propagation, but which would not be expected if the rubber wear resulted mainly from thermal decomposition of the rubber surface region.

The wear rate when a rubber block is sliding on a hard, rough surface is closely related to the friction process. The reason is that the frictional interaction generate strong tensile stresses which will drive crack propagation. In this context we

\footnotetext{
1 The total tread loss is estimated to be about $10^{9} \mathrm{~kg} \mathrm{year}^{-1}$ into particles with a mean effective diameter of about $20 \mu \mathrm{m}$. Thus, one would expect to find tire dust everywhere. However, this is not the case because of relative fast biodegradation (attacks by bacteria and fungi) of the rubber particles (see, e.g., [22]).

2 In addition to tire tread wear resulting from removal of small rubber particles, it has been suggested that gaseous hydrocarbon emission may contribute to tire tread wear. However, experiment have shown (see, e.g., [22]) that the contribution to tire tread wear from gaseous hydrocarbon emission is negligible.
}

note that most real surfaces have roughness on many different length scales (they are typically fractal-like) and large cracks are mainly driven by large asperities as indicated in figure 2 . In addition to large tensile stresses at the exit of asperity contact regions (see figure 2), the frictional work may result in a strong temperature increase in the surface region of the rubber, which has two different effects: (a) the temperature increase makes the rubber more elastic (less viscous), which result in stronger stress concentration at the crack tip and faster crack propagation (this is usually (but equivalently) expressed as a decrease in the energy per unit area, $G(v, T)$, necessary to propagate the crack, as the temperature $T$ at the crack tip increases) $[15,16]$. (b) The temperature increase, if large enough, may result in thermally activated bond-breaking processes everywhere where it is hot enough. Note that this bond-breaking process may occur not just close to crack tips (where thermally activated, stress aided bond breaking occurs [17]) but everywhere. Of course, the probability to break a bond at a crack tip may be higher than elsewhere but many more bonds occur far away from the crack tips, and if the temperature is high enough this 'entropy' effect may dominate resulting in the formation of a thermally decomposed (often 'smear-like') surface layer on the rubber surface. Switching of wear process, from particle removal via crack propagation, to formation of a thermally decomposed rubber surface layer, has indeed been observed for tire tread rubber depending on the severity of the driving condition, see below.

We note that while rubber friction on clean rough surfaces typically vary very little from one substrate surface to another ${ }^{3}$ [18], the wear rate may change by an order of magnitude or more [18]. This can be understood as follows: the friction between a tire and a road surface is mainly due to the timedependent deformation of the rubber by the road surface

\footnotetext{
3 Test performed by the Opel car company.
} 

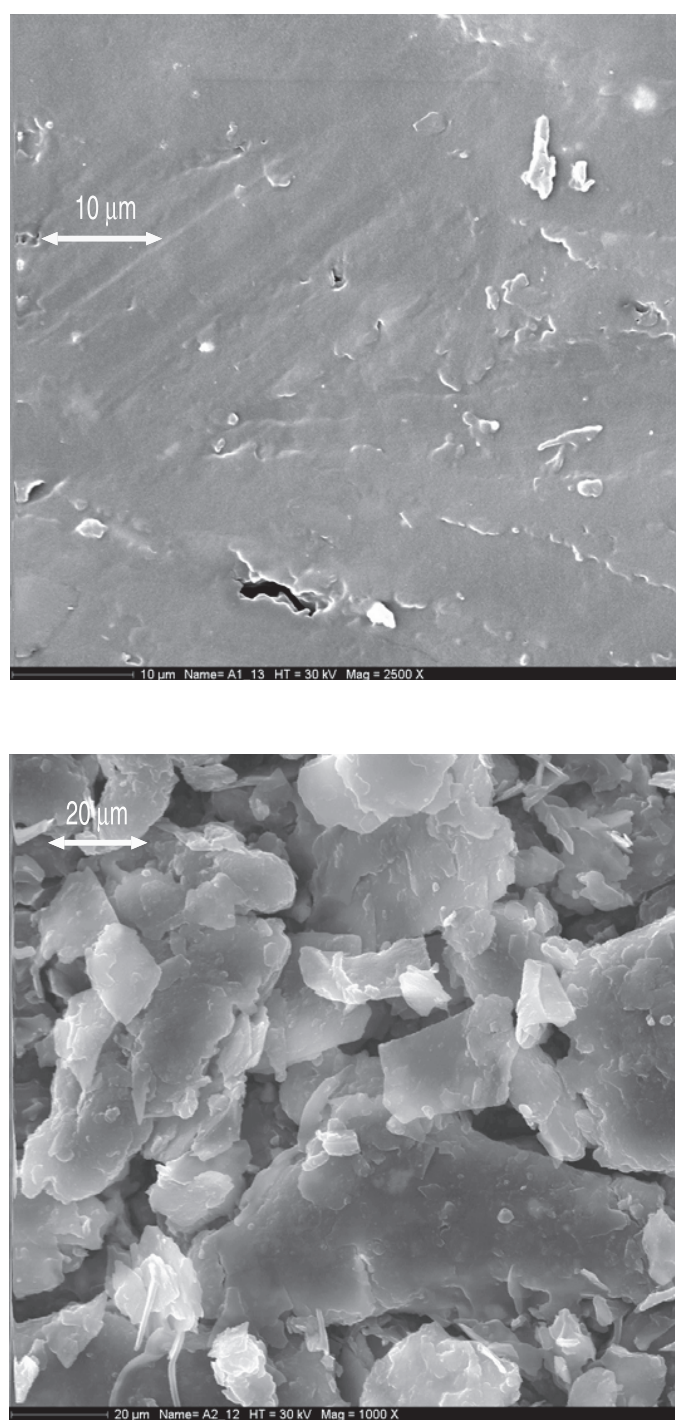

Figure 1. SEM pictures of rubber tread block surface before (top) and after (bottom) use. Courtesy of S Bistac.

asperities. That is, during slip the road asperities will generate time-dependent (pulsating) forces acting on the rubber surface and, because of the internal friction in the rubber, some part of the deformation energy will be transformed into heat. Since road surfaces have surface roughness on a large range of length scales (say, from centimeter to nanometer), the tire-road friction will have contributions from rubber deformations on a wide distribution of length scales.

When the contact between a tire and a road is observed at increasing magnification, the area of real contact will monotonically decrease. At some high magnification, typically corresponding to a resolution (or wavelength $\lambda_{\mathrm{c}}$ ) of order micrometer, the stresses and the temperature increase during slip will be so high that bonds in the rubber will rupture resulting in, e.g. micrometer sized cracks and wear processes. In the theory of rubber friction we have developed, we only take into account the asperity-induced deformation of the rubber down to the length scale $\lambda_{c}$. The range of surface roughness length scales (or wavelength) which contribute to the friction will change from one surface to another in such a
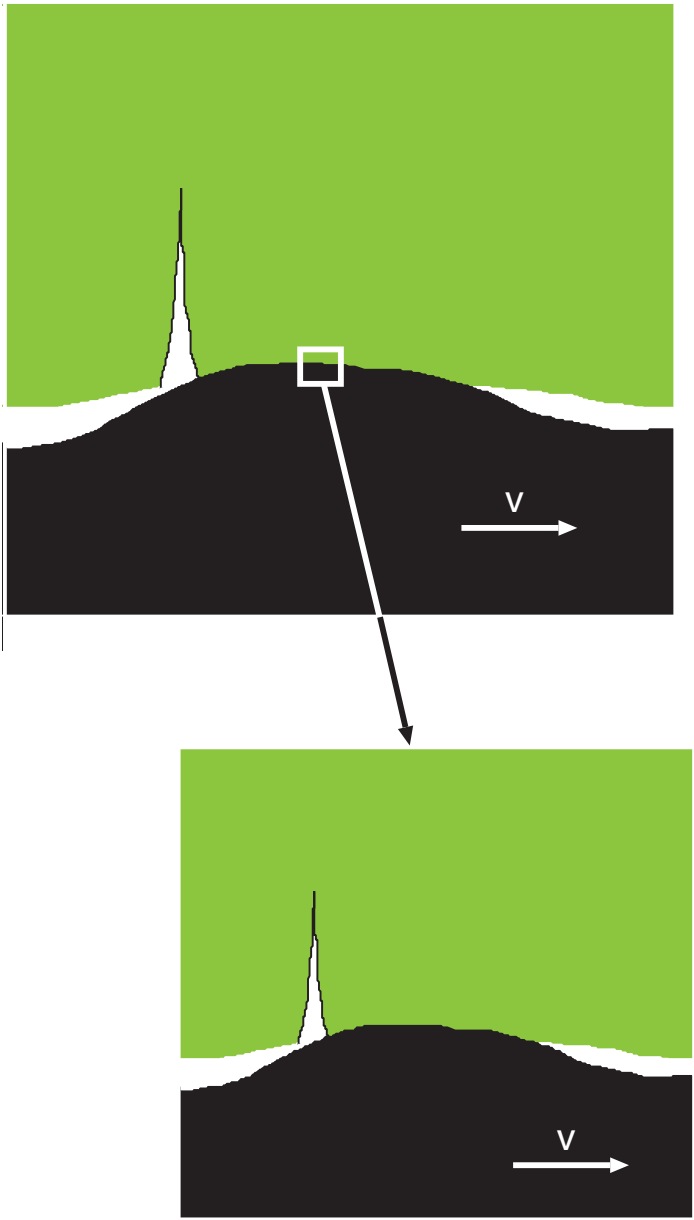

Figure 2. Large asperities will drive the propagation of large cracks while small cracks, observed at higher magnification, are driven mainly by smaller asperities. This follows from the fact that the stress field from an asperity contact region with linear size $d$ will extend into the elastic solid a distance of order $d$.

way that at the resolution $\lambda_{\mathrm{c}}$ the stresses (and the temperature increase) at the rubber surface corresponds to the rupture limit. This theory explains why the tire-road friction varies very little between different (clean and dry) road surfaces (see footnote 3) [18], in spite of large changes in the surface topography and root-mean-square roughness. Thus, a smoother road surface will (in comparison to a surface with larger roughness), in general, result in a smaller cut-off $\lambda_{c}$, and a larger range of surface roughness wavelength components will contribute to the friction, in such a way that there is only a small change in the friction in most cases. On the other hand the predicted wear rate (see below), which depend on $\lambda_{c}$, may change tremendously between different surfaces (it tend to decrease with decreasing surface roughness amplitude, assuming no change in the 'sharpness' of the roughness), which is in good agreement with experimental observations [18].

The results above are for clean surfaces. If the tire surface is contaminated by small particles, then the cut-off length $\lambda_{\mathrm{c}}$ may instead be determined by the (typical) particle diameter $D$. Similarly, if there is a liquid on the road surface the cut-off may be determined by the liquid squeeze-out process. In this 
case the cut-off will depend on the thickness of the fluid layer and on the viscosity of the fluid and on the tire rolling and slip velocities. Thus, in this case the effective cut-off length $\lambda_{c}$ is not determined by the rupture strength of the rubber, and one now expect (and observe) a much larger spread in tire-road friction values between different road surfaces, and usually also smaller tire tread wear ${ }^{4}$ [20].

In this paper I shall present a theory of rubber wear based on the picture presented above. I shall derive the distribution of wear particle sizes $\Phi(D)$ which turns out to be in remarkable good agreement with experiment. I shall also show that the wear rate $\mathrm{d} V / \mathrm{d} L$ (where $V$ is the volume of the rubber removed from a block after sliding the distance $L$ ) is consistent with experimental data for tire tread block wear.

\section{Basic experimental results}

Tire tread wear depends on the driving conditions, and has been studied in great detail for constant slip conditions (see e.g. [19]). Experiments are usually performed with the wheel mounted on the bed of a trailer. The tire can be subjected to various conditions of yaw (tilt), vertical load and brake torque. The wear rate usually increases monotonically as the longitudinal slip or cornering angle increases, and for the longitudinal slip of order $\sim 0.1$ and car velocity $\sim 20 \mathrm{~m} \mathrm{~s}^{-1}$ the wear rate (lost volume of rubber per unit distance moved by the car) $\mathrm{d} V / \mathrm{d} L \approx 10^{-8} \mathrm{~m}^{2}$ or $\sim 10^{-7} \mathrm{~m}^{2}$ per unit slip distance. During normal driving of a passenger car the slip is most of the time much smaller than 0.1 , and the wear volume per unit distance traveled is typically $\sim 10^{-10} \mathrm{~m}^{2}$, corresponding to about $100 \mathrm{mg} \mathrm{km}^{-1}$.

\section{Rubber wear: particle distribution}

Let us consider a rubber block sliding under stationary condition on a rough, hard substrate. Assume that the nominal contact area is $A_{0}$. When steady state is reached, rubber particles will be removed from the rubber block forming some size distribution $\Phi(D)$ with

$$
\int_{0}^{\infty} \mathrm{d} D \Phi(D)=1 .
$$

Let $c(t)$ be the length of a crack into the rubber block from the rubber surface. Let us consider the probability distribution $\Psi(D, t)$ of crack sizes:

$$
\Psi(D, t)=\langle\delta(D-c(t))\rangle
$$

where $\langle\cdots\rangle$ stands for averaging over all the cracks. The rubber surface is exposed to pulsating stresses which drive the cracks.

\footnotetext{
4 Rubber wear experiments performed with abrasive paper have shown that often the wear rate is larger in an oil as compared to dry condition. This may be caused by two effects: (a) if the oil can interdiffuse in the rubber this will result in swelling and reduced internal friction in the rubber. This in turn leads to higher stress concentration, and larger wear even if the sliding friction may be reduced. (b) In an oil rubber wear particles may be effectively removed from the sliding interface (they are dispersed in the oil), while under dry (laboratory) condition they typically attach to the sliding surfaces, forming thin smear-like protective layers which reduces the friction and the wear. See [21] for results illustrating these facts.
}

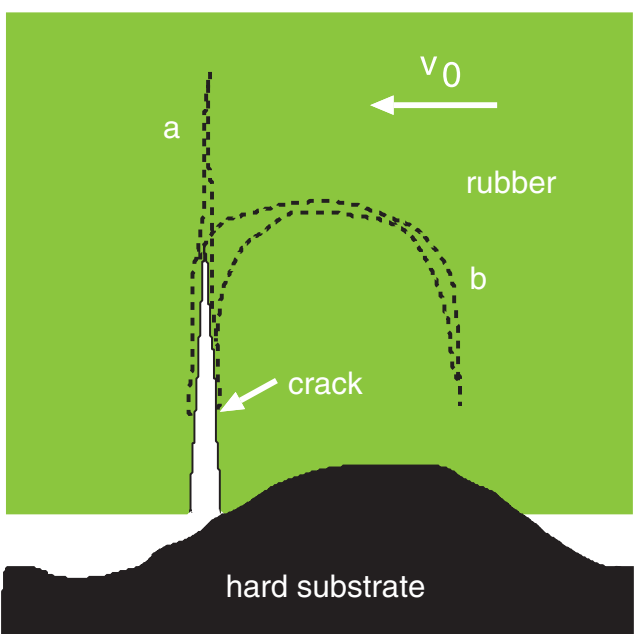

Figure 3. A crack can propagate (mainly) normal to the surface a or 'turn around' $b$, and produce a wear particle. The crack is on the average strait over a distance $l$ (the crack mean free path).

Let $v(D)$ is the average velocity of the crack tip when the crack has the size $D$. We can consider the average as an ensemble average or as the average over many cracks exposed to the same type of (usually pulsating) driving stress field. Using the definition (2) we get

$$
\frac{\partial \Psi}{\partial t}=-\frac{\partial}{\partial D}(\Psi v(D))-\frac{v(D)}{l} \Psi
$$

where $[v(D) / l] \Psi$ is the rate of removal of a particle of size $D$, see figure 3 . We note that a crack can 'turn around' as indicated in figure 3 when the crack tip reaches some defect in the rubber, which could be an agglomeration of filler particles, or a domain of natural rubber (which could strain crystallize when exposed to the strong stress at a crack tip) in a compound consisting of, e.g. styrene-butadiene copolymer and natural rubber. We will refer to $l$ as the crack mean free path.

At stationary state $\partial \Psi / \partial t=0$ giving

$$
\frac{\mathrm{d}}{\mathrm{d} D}(\Psi v(D))+\frac{v(D)}{l} \Psi=0
$$

or

$$
v(D) \Psi=B \mathrm{e}^{-D / l}
$$

where $B$ is a normalization constant. We assume the normalization condition

$$
\int_{0}^{\infty} \mathrm{d} D \Psi(D)=1
$$

which gives

$$
B=\left(\int_{D_{1}}^{\infty} \mathrm{d} D[v(D)]^{-1} \mathrm{e}^{-D / l}\right)^{-1}
$$

where we have introduced a short-distance cut-off length $D_{1}$ (see below). The distribution of wear particles $\Phi(D)$ must be proportional to $v(D) \Psi(D)$ so that, using (1) and (5),

$$
\Phi(D)=l^{-1} \mathrm{e}^{-\left(D-D_{1}\right) / l} .
$$




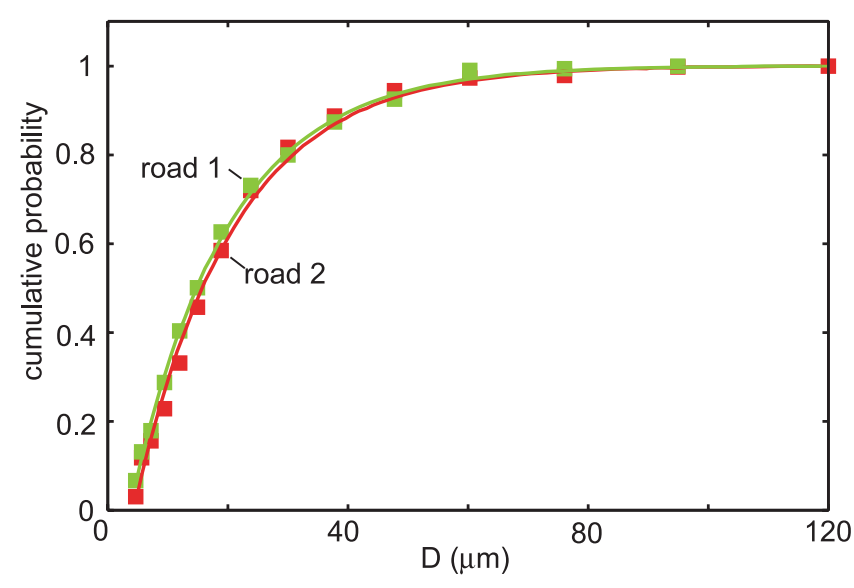

Figure 4. Distribution of rubber wear particles from tire generated during driving on two different highways. About 300 wear particles was studied and the effective diameter $D$ was defined as

$D=(6 V / \pi)^{1 / 3}$, where $V$ is the (measured) volume of the particle. Based on data (table 3) from [22].

Note that the average particle diameter

$$
\bar{D}=\langle D\rangle=\int_{D_{1}}^{\infty} \mathrm{d} D D \Phi(D)=l+D_{1} \approx l
$$

since typically $l \gg D_{1}$. The average volume of a wear particle is

$$
\langle\Delta V\rangle=\frac{\pi}{6} \bar{D}_{V}^{3}=\frac{\pi}{6} \int_{D_{1}}^{\infty} \mathrm{d} D D^{3} \Phi(D) \approx \pi l^{3}
$$

so that $\bar{D}_{V} \approx 1.82 \bar{D}$.

The cumulative probability

$$
\int_{D_{1}}^{D} \mathrm{~d} D^{\prime} \Phi\left(D^{\prime}\right)=1-\mathrm{e}^{-\left(D-D_{1}\right) / l} .
$$

This $D$-dependence is in good agreement with experimental data. Thus, in figure 4 I show the cumulative distribution of wear particle sizes from two road surfaces. The solid lines are the best possible fit to the data using the function (7) with $D_{1}=4.3 \mu \mathrm{m}$ and $l=16.5 \mu \mathrm{m}$ for one road surface, and $D_{1}=3.6 \mu \mathrm{m}$ and $l=16.1 \mu \mathrm{m}$ for another road surface. Note that while $l$ differ by $\sim 2 \%$ between the two cases, $D_{1}$ differ by $\sim 18 \%$. This indicate that $l$ is an intrinsic rubber property, and support our interpretation of $l$ as a crack mean free path, while $D_{1}$ also depend on the road surface. Note that $D_{1}$ is of order $\lambda_{c} / \pi$. Thus, the smallest rubber wear particles will be of order the cut-off length in the rubber friction theory, which depends on the rupture strength of the rubber.

Note that no rubber wear particles with diameter smaller than $D \approx D_{1} \approx 3 \mu \mathrm{m}$ could be detected. This is in accordance with other measurements which show that the smallest rubber wear particles produced from tires on road surfaces is of order a few micrometer $[23,27,28]$. This is also in accordance with the rubber friction theory developed in [24, 25], which shows that the rupture stress of the rubber is typically reached at a magnification corresponding to a length scale of order a few micrometers. Figure 4 also shows that there are no wear particles larger than $D_{0} \approx 120 \mu \mathrm{m}$.

\section{Rubber wear rate}

Consider now a rubber block with a nominally flat surface (surface area $A_{0}$ ) sliding (velocity $v_{0}$ ) on a hard randomly rough substrate surface. If there are $N_{0}$ cracks on the rubber surface area $A_{0}$ then the volume of removed rubber per unit time is

$$
\frac{\mathrm{d} V}{\mathrm{~d} t}=N_{0} \int \mathrm{d} D \frac{\pi D^{3}}{6} \frac{v(D)}{l} \Psi(D) .
$$

Assuming $N_{0}=\xi A_{0} / D_{1}^{2}$, where $\xi$ is a number of order unity, we get

$$
\frac{\mathrm{d} V}{\mathrm{~d} t}=C A_{0} v_{0}
$$

where

$$
C=\frac{\pi \xi D_{1}}{6 l v_{0}} \int_{D_{1}}^{\infty} \mathrm{d} D\left(\frac{D}{D_{1}}\right)^{3} v(D) \Psi(D) .
$$

If we use (5) we get

$$
C=\frac{\pi \xi D_{1} B}{6 l v_{0}} \int_{D_{1}}^{\infty} \mathrm{d} D\left(\frac{D}{D_{1}}\right)^{3} \mathrm{e}^{-D / l} .
$$

Substituting $B$ from (6) into this equation, and introducing the sliding distance $L=v_{0} t$ we get

$$
\frac{\mathrm{d} V}{\mathrm{~d} L}=C A_{0}
$$

where

$$
C=\frac{\pi \xi D_{1}}{6 l} \frac{\int_{D_{1}}^{\infty} \mathrm{d} D\left(D / D_{1}\right)^{3} \mathrm{e}^{-D / l}}{\int_{D_{1}}^{\infty} \mathrm{d} D\left[v_{0} / P(\zeta) v(D)\right] \mathrm{e}^{-D / l}} .
$$

In (8) I have introduced the factor $P(\zeta)=A(\zeta) / A_{0}$ which is the ratio between the rubber-substrate contact area at the magnification $\zeta$ and the nominal contact area $A_{0}$. Thus, the (average) crack tip velocity $v(D)$ in (8) is now not the average over the whole contact area but only over the (apparent) area of contact observed at the magnification $\zeta$.

Since typically $l \gg D_{1}$ we get

$$
\int_{D_{1}}^{\infty} \mathrm{d} D\left(\frac{D}{D_{1}}\right)^{3} \mathrm{e}^{-D / l} \approx \frac{6 l^{4}}{D_{1}^{3}} \mathrm{e}^{-D_{1} / l} .
$$

Substituting this in (8) gives

$$
C=\frac{\pi \xi l^{3}}{D_{1}^{2}}\left(\int_{D_{1}}^{\infty} \mathrm{d} D \frac{v_{0}}{P(\zeta) v(D)} \mathrm{e}^{-\left(D-D_{1}\right) / l}\right)^{-1}
$$

Consider the contact between the rubber and the countersurface at the magnification $\zeta$. In the asperity contact regions time-dependent deformations of the rubber occur, at the characteristic frequency $\omega=\zeta q_{0} v_{0}$, where $q_{0}$ is a reference wavevector (see below), and where $v_{0}$ is the sliding velocity. We can relate the particle size $D$ to the magnification $\zeta$ as follows. At the magnification $\zeta$ one observe surface wavelength roughness with the wavevector $q=q_{0} \zeta$ where $q_{0}$ is some suitable chosen reference wavevector, usually chosen to be the long-wavelength roll-off wavevector of the 
surface roughness power spectra. Surface roughness with the wavevector $q$ gives rise to a stress field which extend $\sim 1 / q$ into the solid so we identify $D \approx 1 / q=1 /\left(q_{0} \zeta\right)$.

If $T$ is the period of the pulsating deformations which a crack of size $D$ is exposed to, then $v_{0} T=2 \pi D$ and $v(D) T=$ $\delta c(D)$, where $\delta c(D)$ is the increase in the crack length during one period of deformation. Thus $v(D) / v_{0}=\delta c(D) / 2 \pi D$ and (10) becomes

$$
C=\frac{\xi l^{3}}{2 D_{1}^{3}}\left(\int_{D_{1}}^{\infty} \mathrm{d} D \frac{D}{D_{1}} \frac{1}{P(\zeta) \delta c(D)} \mathrm{e}^{-\left(D-D_{1}\right) / l}\right)^{-1} .
$$

Let us define the average

$$
\frac{1}{\langle P \delta c\rangle}=\frac{D_{1}}{l^{2}} \int_{D_{1}}^{\infty} \mathrm{d} D \frac{D}{D_{1}} \frac{1}{P(\zeta) \delta c(D)} \mathrm{e}^{-\left(D-D_{1}\right) / l} .
$$

Note that if $P \delta c$ would be independent of $D$ then $\langle P \delta c\rangle=$ $P \delta c$ where we again have assumed $l \gg D_{1}$. Using (9)-(11) gives

$$
C=\frac{\xi}{2} \frac{\langle P \delta c\rangle}{D_{1}} \frac{l}{D_{1}}
$$

In a typical case $P \approx 0.05, D_{1} \approx 3 \mu \mathrm{m}, l / D_{1} \approx 4, A_{0} \approx$ $0.01 \mathrm{~m}^{2}$, and for the slip velocity of order $\sim 1 \mathrm{~m} \mathrm{~s}^{-1}$ one have for (a typical) crack length of order $\sim 10 \mu \mathrm{m}, \delta c \approx 10^{-10} \mathrm{~m}$ (see below) giving $\mathrm{d} V / \mathrm{d} L=C A_{0} \approx 10^{-7} \mathrm{~m}^{2}$ which is similar to the observed value of $\mathrm{d} V / \mathrm{d} L$. Thus, the theory does not only predict the correct distribution of wear particle sizes, but also the calculated wear rate is consistent with experimental observation. However, an accurate treatment of tire tread wear must take into account the non-uniform slip of the tread blocks which occurs during cornering or ABS braking. In particular, the velocity of the car $v_{\mathrm{c}}$ is not equal to the slip velocity $v_{0}$.

The tire rubber wear volume per unit distance $L=v_{\mathrm{c}} t$ during cornering or braking (with car velocity $v_{\mathrm{c}}$ ) is given by

$$
\frac{\mathrm{d} V}{\mathrm{~d} L}=\langle C\rangle A_{0}
$$

where $A_{0}$ is the rubber surface area in apparent contact with the road surface (which is approximately given by the ratio between the load $F_{\mathrm{N}}$ acting on the tire, and the gas pressure $p_{0}$ in the tire), and where

$$
\langle C\rangle=\frac{\pi \xi l^{2}}{D_{1}^{2}}\left\langle\left(\int_{D_{1}}^{\infty} \frac{\mathrm{d} D}{l} \frac{v_{\mathrm{c}}}{P(\zeta) v(D)} \mathrm{e}^{-\left(D-D_{1}\right) / l}\right)^{-1}\right\rangle
$$

where $\langle\cdots\rangle$ stands for the average over all the tread blocks in the tire-road foot print, and average over time. We note that both $P(\zeta)$ and $v(D)$ depends on the location of the tread block in the foot print area. In particular, close to the exit of the footprint the tread block slip velocity is maximal which may result in a strong temperature increase of the rubber in the asperity contact regions, which will result in a strong increase in the crack tip velocity $v(D)$ (which, according to (14), will strongly increase the wear rate) and will also influence the relative contact area $P(\zeta)$ somewhat. The temperature increase will also increase as the slip increases, and this is the main reason for why rubber wear increases with increasing slip [19]. We note that for rubber which does not

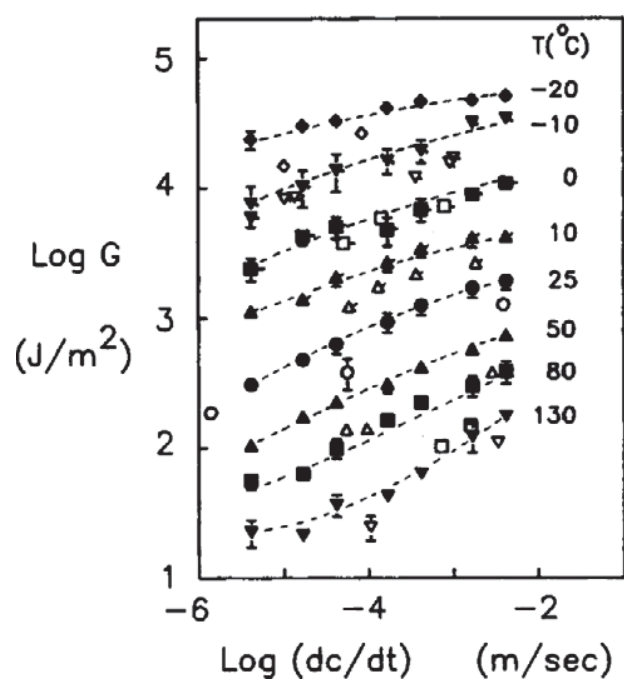

Figure 5. The fracture energy (per unit area) for SB-rubber crosslinked with $1.5 \%$ peroxide. Reproduced with permission from [27]. Copyright 1993 Wiley Interscience.

undergo strain crystallization, the dependence of the crack tip velocity $v(D)$ on the temperature and the energy release rate $G$ can be calculated (or estimated) using the theory developed in $[15,16]$.

Neglecting the influence of ozone, the crack tip velocity $v(D) \approx 0$ if the energy release rate $G<G_{0} \approx 30 \mathrm{~N} \mathrm{~m}^{-1}$, while $v(D)$ increases rapidly for $G>G_{0}$. For stationary crack propagation the function $v(D)=F(G)$ has been studied both experimentally [23] and theoretically [15, 16] for viscoelastic solids. No accurate theoretical study exist for non-stationary crack propagation, but for cracks exposed to oscillatory stresses, experimental information exist for how $\delta c$ depends on the (amplitude of) the strain energy release rate $G$. For rubber which does not strain crystallize $\delta c \approx v(D) T$, where $T$ is the period of the oscillating force [16]. In the present context [26] $G \approx \sigma^{2}(\zeta) D / 2|E(\omega)|$ where $\sigma(\zeta)$ is the frictional stress in the contact areas when the system is studied at the magnification $\zeta$. We have $\sigma(\zeta) A(\zeta)=\mu\left(v_{0}\right) p_{0} A_{0}$, where $p_{0}$ is the normal stress or pressure. Thus, $\sigma(\zeta)=$ $\mu\left(v_{0}\right) p_{0} / P(\zeta)$ so that

$$
G \approx \frac{\left(\mu\left(v_{0}\right) p_{0}\right)^{2} D}{2 P^{2}(\zeta)|E(\omega)|}=\frac{\left(\mu\left(v_{0}\right) p_{0}\right)^{2}}{2 \zeta q_{0} P^{2}(\zeta)\left|E\left(q_{0} \zeta v_{0}\right)\right|}
$$

Using the rubber friction and tire model theory developed by Persson [24, 25] we calculate for asphalt road surfaces typically $G \approx 100 \mathrm{~J} \mathrm{~m}^{-2}$. Using the measured results for the velocity and temperature dependence of $G(v, T)$ shown in figure 5 we get for $G \approx 100 \mathrm{~J} \mathrm{~m}^{-2}$, and for the (tire) temperature $T \approx 80^{\circ} \mathrm{C}, \mathrm{d} c / \mathrm{d} t \approx 10^{-4} \mathrm{~m} \mathrm{~s}^{-1}$ giving $\delta c \approx$ $10^{-9} \mathrm{~m}$. Using (13) this gives a wear rate a factor of $\sim 10$ larger than observed experimentally, but we believe this is due to the fact that the (experimental) relation $G(v, T)$ shown in figure 5 is for unfilled SB-rubber. For SB-rubber with carbon filler one expect a larger internal damping in the rubber, which result in an increased energy dissipation close to the crack tip, and to slower crack propagation. Indeed, fatigue crack propagation studies by Klüppel [13, 14] for SB-rubber show that the 


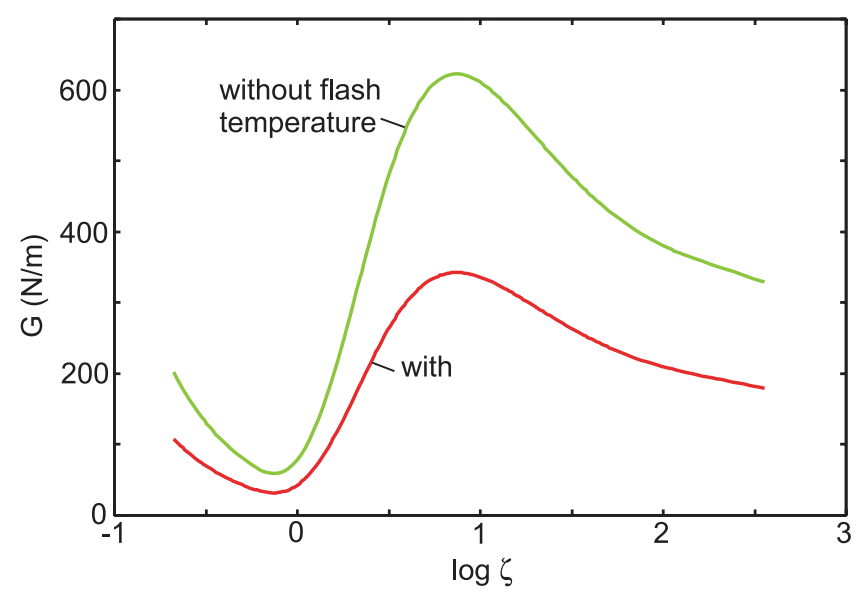

Figure 6. The energy release rate $G(\zeta)$ both with and without including the local temperature increase (flash temperature). The results are for a block of a standard rubber tread compound sliding at $v_{0}=1 \mathrm{~m} \mathrm{~s}^{-1}$ on an asphalt road surface. The magnification $\zeta=1$ corresponds to the wavevector $q_{0}=300 \mathrm{~m}^{-1}$, and the highest magnification to $q_{1}=1.07 \times 10^{5} \mathrm{~m}^{-1}$. The road rms roughness value is $0.78 \mathrm{~mm}$. The maximal kinetic friction is calculated to be $\mu_{\max }=1.0$, and occur for the slip velocity $0.03 \mathrm{~m} \mathrm{~s}^{-1}$.

effective crack tip propagation velocity decreases by about one order of magnitude as the carbon filler concentration increases from 0 to $\sim 20 \%$. These studies was performed at room temperature and for low-frequency $(4 \mathrm{~Hz})$ pulsating applied stress, which differ from the condition in tire applications, but this should not affect the qualitative conclusion that including filler will reduce the crack propagation velocity and make the predicted wear rate closer to experimental observations.

When the tire temperature increases the wear rate usually increases strongly. This is due to the reduction in the internal damping in the rubber, and consequently strong increase in the crack tip propagation velocity (see, e.g., figure 5). This effect is well documented experimentally, e.g., studies by S A Bridgestone (2002), reported on in [29], show a decrease in the tire life for truck tires by a factor of $\sim 5$ as the environmental temperature increases from 5 to $40^{\circ} \mathrm{C}$.

In figure 6 we show the energy release rate $G(\zeta)$ both with and without including the local temperature increase (flash temperature). The results are for a block of a standard rubber tread compound sliding at $v_{0}=1 \mathrm{~m} \mathrm{~s}^{-1}$ on an asphalt road surface. In the calculation we have used (16) and a simple model for the tire based on the rubber friction theory described in [25]. Note that $G>G_{0}$ and crack propagation should contribute to the rubber wear.

From equation (16) it is easy to understand the qualitative form of the $G$-curve in figure 6 . As the magnification $\zeta$ decreases from its maximum value $\zeta_{1}$, the size-parameter $D \sim 1 / \zeta$ and the (apparent) relative contact area $P(\zeta)$ both increases, while the magnitude of the viscoelastic modulus $|E(\omega)|$ decreases. Initially, for large $\zeta$, the increase in $D /|E|$ dominates over the decrease in $P^{-2}$, resulting in the observed increase in $G$ with decreasing magnification. However, at small enough magnification (typically $\zeta \approx 10$ ) the contact area very rapidly increases with decreasing magnification (see e.g., $[16,24,25])$, and now the decrease in $P^{-2}$ is larger

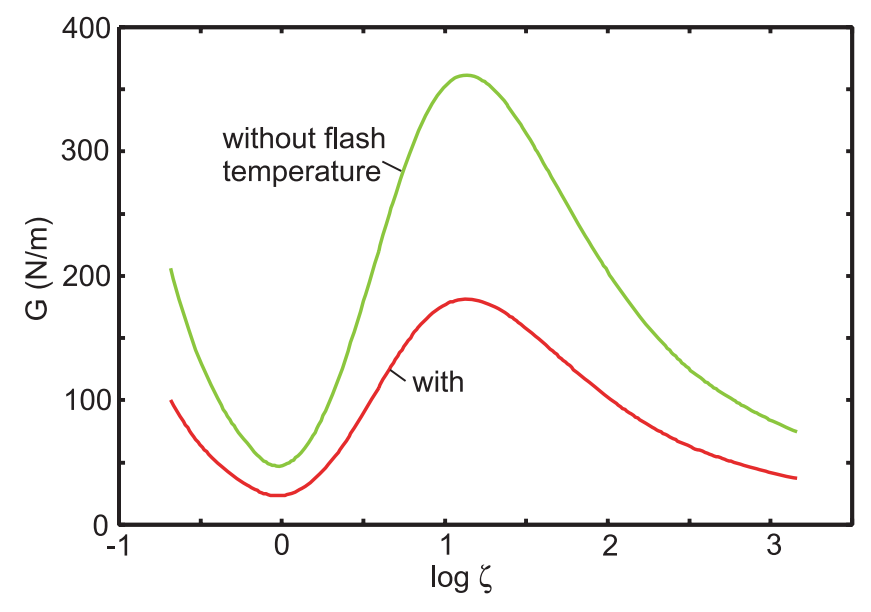

Figure 7. The energy release rate $G(\zeta)$ both with and without including the local temperature increase (flash temperature). The results are for a block of a standard rubber tread compound sliding at $v_{0}=1 \mathrm{~m} \mathrm{~s}^{-1}$ on an asphalt road surface. The magnification $\zeta=1$ corresponds to the wavevector $q_{0}=300 \mathrm{~m}^{-1}$, and the highest magnification to $q_{1}=4.3 \times 10^{5} \mathrm{~m}^{-1}$. The road $\mathrm{rms}$ roughness value is $0.51 \mathrm{~mm}$. The maximal kinetic friction is calculated to be $\mu_{\max }=0.99$, and occur for the slip velocity $0.027 \mathrm{~m} \mathrm{~s}^{-1}$.

than the increase in $D /|E|$, resulting in a decreasing $G$ with decreasing magnification. Finally, at low enough magnification the (apparent) relative contact area approach the nominal contact area and $P \rightarrow 1$ and the $G$-function will increase again with decreasing magnification due to the dependence of $D /|E|$ on the magnification.

In figure $7 \mathrm{I}$ show the same results for another smoother asphalt road surface. In this case $G$ is of order $G_{0}$. However, since the results of $G$ given in these figures represent average values, there will in actual fact be some places where $G$ will be larger. Thus we conclude that on both surfaces the surface roughness induced stresses should be able to propagate surface cracks. However, it is clear that on the smoother asphalt road surface $G$ is smaller than on the rougher surface, and this will lead to much smaller (average) $\delta c$-values on the smoother surface. Concerning the wear rate, the factor $1 / D_{1}^{2}$ in (13) will increase the wear rate on the smoother surface by a factor of $\sim 16$ but this is overcompensated by the strong reduction in $\delta c$ which may be of order $\sim 50$. Thus the net result is a smaller wear rate on the smoother asphalt surface.

\section{Discussion}

One can distinguish between at least three rubber wear processes.

(A) Rubber wear resulting from the formation and (slow) propagation of cracks. This is the mechanism considered above, and probably the most common tire tread wear process on surfaces with blunt surface roughness.

(B) Rubber wear on substrates with very sharp surface roughness, e.g. sand paper or safety walk, seems to be a cutting process, where the sharp substrate asperities cut away rubber fragments from the rubber surface. This involves relative fast crack propagation where the bond 


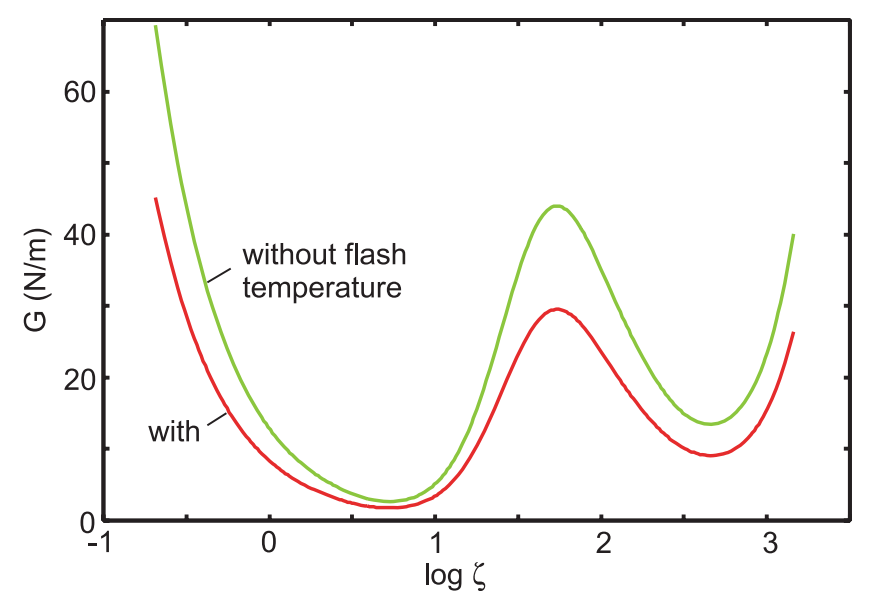

Figure 8. The energy release rate $G(\zeta)$ both with and without including the local temperature increase (flash temperature). The results are for a block of a standard rubber tread compound sliding at $v_{0}=1 \mathrm{~m} \mathrm{~s}^{-1}$ on a sandpaper (grit 180). The magnification $\zeta=1$ corresponds to the wavevector $q_{0}=1000 \mathrm{~m}^{-1}$, and the highest magnification to $q_{1}=1.44 \times 10^{6} \mathrm{~m}^{-1}$. The sandpaper rms roughness value is $0.03 \mathrm{~mm}$. The maximal kinetic friction is calculated to be $\mu_{\max }=1.16$, and occur for the slip velocity $0.174 \mathrm{~m} \mathrm{~s}^{-1}$.

breaking at the crack tips is facilitated by sharp substrate asperities, similarly to experimental studies of crack propagation where the sharp edge of a razor blade was pushed against the crack edge [28]. In this case the rubber wear volume is proportional to the applied load and to the sliding distance but only weakly dependent on the sliding velocity. In this case without the direct influence on the bond breaking by the sharp asperities at the crack tips, crack propagation is unlikely to contribute to the wear rate since the $G(\zeta)$-function is typically much smaller than $G_{0}$; see figure 8 for an example (calculated result), where a tread rubber is sliding with $v_{0}=1 \mathrm{~m} \mathrm{~s}^{-1}$ on a sand paper 180 substrate. In this case $G<G_{0}$ for $\zeta>1$ and no crack propagation is expected without the direct influence by the asperities on the bond-breaking process at the crack tip. However, the rubber wear rate on sandpaper 180 is typically 10 times (or more) higher than on asphalt road surfaces. We attribute this to cutting by the sharp surface asperities on the sandpaper surface.

(C) Thermal and stress induced rubber surface modifications may result in a thin liquid-like smear film on the rubber (and substrate) surface. This can occur for the same roadrubber system as in (A) but under different operational conditions. As an example, we show in figure 9 the surface topography for the same tire-road system as in figure 1 but under different operational conditions. Thus, the surface shown in figure 1 (bottom) is from a tread block taken from the inner tire of a car which was driven at high speed along a circular track. Figure 9 shows the surface from a tread block taken from the outer tire. Because of load transfer (resulting from the centrifugal force) the load on the outer tires was several times higher than on the inner tire. This resulted in a much higher rubber temperature and a longer tire-road footprint (which will influence the tire tread slip dynamics). As a consequence, a thin smear

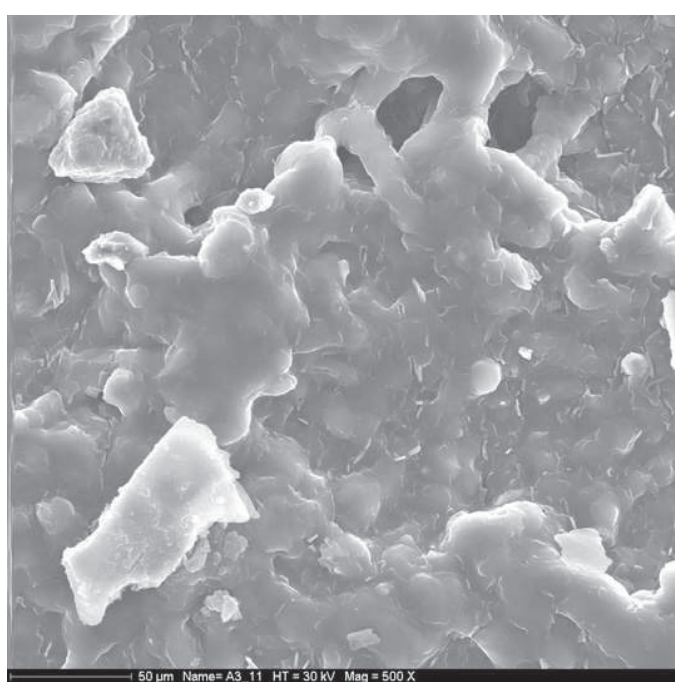

Figure 9. SEM surface topography picture of tread block from outer tire of a car driven at high speed (large centrifugal force) along a circular path. Courtesy of S Bistac.

layer seems to have formed on the tire tread surface. Such smear layers can strongly reduce the wear rate by protecting the underlying rubber. The smear films seems to be related to mechanical rupture of macromolecules followed by oxidation processes; experiment have shown that in an inert atmosphere no smear film is formed and the rubber wear occur by the formation of dry rubber particles (process (A)).

It is clear from (13) that reducing the crack mean free path $l$ will reduce the wear rate. We note that if $\langle P \delta c\rangle$ is independent of $l$ (which is the case if $P \delta c$ does not depend on the size $D$ of the crack), then $C \sim l$ which just reflect that the surface area (formed by crack propagation), of a rubber wear particle with linear size $l$, scales as $l^{2}$, while the particle volume scales as $l^{3}$. The volume-to-surface ratio scales as $\sim l$, which is the origin of the factor of $l$ in (13).

Unfilled rubber compounds have very bad wear resistance, and this is, at least in part, due to the fact that there are no (strong) inhomogeneities which can scatter the crack tip, and reduce the crack mean free path. There are several ways to reduce the crack mean free path $l$.

(a) Adding filler particles such as carbon black or silica particles will strongly reduce the wear. The filler particles form clusters of various sizes, and when the crack tip reach a particle cluster it may bend by $\sim 90^{\circ}$ rather than penetrate through the particle cluster [30]. This will only happen if the rubber molecules are strongly enough bound to the particle cluster so that the stress field from the crack tip cannot easily break and penetrate the particle cluster. This seems to be the case for carbon black (probably because of the large surface area of the fractal-like particles) but not for untreated silica particles, which gives much smaller reduction in the wear as compared to carbon particles. However, the silica filler particles used for tire tread applications are coated by monolayers of grafted 
molecules which can bind strongly to the rubber matrix, and these particles result in a similar strong reduction of the rubber wear as compared to carbon particles. In order to reduce the crack mean free path $l$ the cluster of filler particles must be relative uniformly dispersed: the crack mean free path will be of order the distance between the clusters. Thus, a low concentration of large clusters will not give rise to good wear resistance. Rather, in order to limit the wear particle sizes to a few micrometer, the average distance between the clusters should be of order a few micrometer. At the same time the size of the clusters must be of order micrometer since it is clear that a cluster much smaller than the linear size of a crack will not be able to perturb the path of the crack tip. The two conditions above can only be realized at high filler concentration, and this may be one of the reason for why the volume filling factor of the particles in a typical tire tread compound is of order $\sim 35 \%$.

(b) It is known that adding some natural rubber to styrenebutadiene rubber may reduce the wear rate and generate tread surfaces which are much smoother than for SBrubber without natural rubber, indicating much smaller wear particles. This result can be explained if one notice that the natural rubber component form small domains (diameter of order $1 \mu \mathrm{m}$ or smaller) dispersed in the SB matrix; when the crack tip reaches a domain of natural rubber the latter may strain crystallize, resulting in an obstacle which the crack cannot penetrate. This may again result in a crack which turns around as in figure 3 , and hence to a reduced crack mean free path $l$ and to reduced tire tread wear.

\section{Summary and conclusion}

Rubber wear typically involves the removal of small rubber particles from the rubber surface. For not too sharp roughness, e.g. most road surfaces, this involves crack propagation. I have presented a theory of powdery rubber wear, and derived the distribution of wear particle sizes $\Phi(D)$, which is in excellent agreement with experiment. I have show that the wear rate is consistent with experimental data for tire tread block wear. The theory shows that one way to reduce tire tread wear is to reduce as much as possible the crack tip mean free path $l$. The present theory can be implemented in tire models, where the slip-motion of each individual tread block is accounted for, to predict tire wear. Work along these lines are in progress and will be reported on elsewhere.

\section{Acknowledgments}

I thank Pirelli Pneumatici for support and Oliver Albohr (Pirelli Germany) and Ugo Tartaglino (Pirelli Milan) for useful comments on the paper.

\section{References}

[1] Southern E and Thomas A G 1978 Plast. Rubber Mater. Appl. 3133

[2] Grosch K A and Schallamach A 1965 Trans. Inst. Rubber Ind. 41 T80

[3] Veith A G 1973 Rubber Chem. Technol. 46801

[4] Veith A G and Oblizajek K L 1986 Tire Sci. Technol. 14201

[5] Veith A G and Chirico C E 1979 Rubber Chem. Technol. 52 748

[6] Veith A G 1992 Rubber Chem. Technol. 65601

[7] Veith A G 1987 Polym. Test. 7177

[8] Gough V E 1958/59 Wear 2107

[9] Grosch K A and Schallamach A 1961 Wear 4356

[10] Grosch K A and Turner D M 1960 Wear 31

[11] Cadle S H and Williams R L 1978 Rubber Chem. Technol. 517

[12] Cadle S H and Williams R L 1979 Rubber Chem. Technol. 52 147

[13] Klüppel M 2009 Marcomol. Mater. Eng. 294130

[14] Klüppel M 2009 J. Phys.: Condens. Matter 21035104

[15] Persson B N J and Brener E 2005 Phys. Rev. E 71036123

[16] See, e.g. Persson B N J, Albohr O, Heinrich G and Ueba H 2005 J. Phys.: Condens. Matter 17 R1071

[17] Carbone G and Persson B N J 2005 Phys. Rev. Lett. 95114301 Carbone G and Persson B N J 2005 Eur. Phys. J. E 17261

[18] Westermann S 2007 private communication (Luxembourg: Goodyear Tire Company)

[19] See, e.g. 1995 NASA Technical Memorandum 110186 NASA Tire/Runway Friction Project http://ntrs.nasa.gov/archive/ nasa/casi.ntrs.nasa.gov/19960003383_1996103383.pdf June

[20] Mofidi M, Prakash B, Persson B N J and Albohr O 2008 J. Phys.: Condens. Matter 208

[21] Mofidi M and Prakash B 2008 Proc. Inst. Mech. Eng. J 222667 Mofidi M, Kassfeldt E and Prakash B 2008 Tribol. Int. 41860

[22] Dannis M L 1974 Rubber Chem. Technol. 471011

[23] Gent A N and Pulford C T R 1983 J. Appl. Polym. Sci. 28943

[24] Persson B N J 2001 J. Chem. Phys. 1153840

[25] Persson B N J 2006 J. Phys.: Condens. Matter 187789

[26] Benuzzi D, Bormetti E and Donzella G 2003 Theor. Appl. Fract. Mech. 4055

[27] Gent A N and Lai S-M 1994 J. Polym. Sci. B 321543

[28] Gent A N 1996 Langmuir 124492

[29] Netscher N W et al 2008 A review of patents in tyre cooling Recent Pat. Eng. 287 http://espace.uq.edu.au/eserv/UQ: 151482/Hooman-Patent_Journal_Article.pdf

[30] Gent A N 2006 The Pneumatic Tire ed A N Gent and J D Walter, US Department of Transportation (www.nhtsa.dot.gov) 